\section{TNF inhibition for immune checkpoint inhibitor-induced irAEs: the jury is still out}

\author{
Karijn P. M. Suijkerbuijk (D) and Rik J. Verheijden (D)
}

We congratulate Chen and colleagues on their elegant Review discussing the complex interactions between TNF inhibition and immune checkpoint inhibition (Chen, A. Y., Wolchok, J. D. \& Bass, A. R. TNF in the era of immune checkpoint inhibitors: friend or foe? Nat. Rev. Rheumatol. 17, 213-223 (2021)) ${ }^{1}$. Supported by preclinical data, the authors conclude that short-term TNF inhibition to treat immune checking inhibitor (ICI)induced toxicity should not compromise ICI efficacy. The authors refer to our study examining the effect of immunosuppressive management of ICI-induced toxicity on survival among patients with grade $\geq 3$ immune-related adverse events (irAEs) ${ }^{2}$. In this study, we reported a shorter overall survival in patients who received anti-TNF therapy compared with patients treated with corticosteroids only.

Chen and colleagues advocate that this observation might have been biased owing to the use of overall survival as an outcome measure, as some severe irAEs (such as colitis) have a higher mortality than others (such as endocrine toxicity). However, the overall survival did not differ notably between patients with colitis and patients with other irAEs (adjusted HR 1.15; 95\% CI 0.82-1.60) $)^{2}$. Moreover, there was no difference in toxicity-related mortality between those patients who received anti-TNF therapy and those who did not ${ }^{2}$. Similarly, melanoma-specific survival was shorter in patients who received TNF inhibition ${ }^{2}$.

Chen and colleagues validly comment that we did not account for time to irAE onset, which could have biased the results if the onset of irAEs occurred earlier in patients who received TNF inhibition than in the patients who only received corticosteroids. However, given that these analyses were performed in patients with grade $\geq 3$ irAEs only, we do not expect the differences in time to irAE onset to be notable.

In their Review, the authors mention two small studies that seemingly contradict our findings ${ }^{3,4}$. In 27 ICI-treated patients with melanoma who received anti-TNF therapy for colitis, Lesage et al. reported a median progression free survival (PFS) of 3 months $^{3}$, which was comparable to the
PFS reported for patients being treated with a CTLA4 inhibitor in the CheckMate 067 trial $^{5}$. However, one-third of the patients reported on by Lesage et al. ${ }^{3}$ received a PD1 inhibitor or combination ICI therapy, for which the median PFS in the CheckMate 067 study was considerably higher (7 and 11.5 months, respectively $)^{5}$. In the second study, among patients with ICI-induced colitis, Wang and colleagues observed no difference in overall survival in the patients treated with corticosteroids and an anti-TNF therapy $(n=23)$ and the patients treated with corticosteroids alone $(n=38)^{4}$. Remarkably, preliminary results of a study of 150 patients with ICI-induced colitis from the same institution showed a worse overall survival in those patients who received TNF inhibition compared with those who received vedolizumab (a gut-selective integrin inhibitor; $P=0.042)^{6}$.

In conclusion, we support the authors' hypothesis that the net effect of TNF inhibition on tumorigenesis might be positive or

\section{Anne R. Bass (D) and Allen Y. Chen}

We would like to thank Karijn Suijkerbuijk and Rik Verheijden for their correspondence (Suijkerbuijk, K. P. M. \& Verheijden, R. J. TNF inhibition for immune checkpoint inhibitor-induced irAEs: the jury is still out. Nat. Rev. Rheumatol. https://doi.org/10.1038/ s41584-021-00640-z (2021)) ${ }^{1}$ on our Review (Chen, A. Y., Wolchok, J. D. \& Bass, A. R. TNF in the era of immune checkpoint inhibitors: friend or foe? Nat. Rev. Rheumatol. 17, 213-223 $(2021))^{2}$, which raises some important issues concerning our analysis of their study assessing the safety of TNF inhibitor treatment for immune-related adverse events (irAEs) from immune checkpoint inhibitors (ICIs).

In our Review we wrote, "the use of overall survival as the end point might have introduced negative in different situations. Nevertheless, we think that more research is needed before we can rule out detrimental effects of TNF inhibition for irAEs.

There is a reply to this letter by Bass, A. R \& Chen, A. Y. Nat. Rev. Rheumatol. https:// doi.org/10.1038/s41584-021-00641-y (2021).

Karijn P. M. Suijkerbuijk (DD ${ }^{凶}$ and Rik J. Verheijden (D) Department of Medical Oncology, UMC Utrecht, Utrecht, The Netherlands. 凶e-mail: K.Suijkerbuijk@umcutrecht.nl https://doi.org/10.1038/s41584-021-00640-z

1. Chen, A. Y., Wolchok, J. D. \& Bass, A. R. TNF in the era of immune checkpoint inhibitors: friend or foe? Nat. Rev. Rheumatol. 17, 213-223 (2021).

2. Verheijden, R. J. et al. Association of anti-TNF with decreased survival in steroid refractory ipilimumab and anti-PD1 treated patients in the Dutch Melanoma Treatment Registry. Clin. Cancer Res. 26, 2268-2274 (2020).

3. Lesage, $\mathrm{C}$. et al. Incidence and clinical impact of anti-TNF $\alpha$ treatment of severe immune checkpoint inhibitor-induced colitis in advanced melanoma: the mecolit survey. J. Immunother. 42, 175-179 (2019).

4. Wang, Y. et al. Immune-checkpoint inhibitor-induced diarrhea and colitis in patients with advanced malignancies: retrospective review at MD Anderson. J. Immunother. Cancer 6, 37 (2018).

5. Wolchok, J. D. et al. Overall survival with combined nivolumab and ipilimumab in advanced melanoma. N. Engl. J. Med. 377, 1345-1356 (2017).

6. Zou, F. et al. S0137 Comparative study of vedolizumab and infliximab treatment in patients with immunemediated diarrhea and Colitis. Am. J. Gastroenterol. 115, S68 (2020)

\section{Competing interests}

K.P.M.S. reports receiving speakers bureau honoraria from Novartis and Roche, and is a consultant and/or advisory board member for Novartis, Roche, MSD, Abbvie, Pierre Fabre and Bristol-Myers Squibb. R.J.V. declares no competing interests.

\title{
Reply to: TNF inhibition for immune checkpoint inhibitor-induced irAEs: the jury is still out
}

confounders as some high-grade irAEs (such as colitis) have a higher mortality than others (such as high-grade endocrine toxicity, which can be treated with hormone replace-

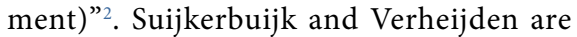
correct in pointing out that melanomaspecific survival was also shorter in the TNF inhibitor-treated patients, suggesting that toxicity-related deaths did not explain the differences in survival. We agree, this omission was an oversight on our part.

We do believe, however, that unmeasured confounders impacted their analysis of TNF inhibitor-related mortality, particularly as most TNF inhibitor-treated patients in their study received ipilimumab (an anti-CTLA4 therapy) $)^{3}$. The study analysed patients 